\title{
On the Stability of Thermonuclear Burning Fronts in Type Ia Supernovae
}

\author{
F.K. Röpke ${ }^{1}$ and W. Hillebrandt ${ }^{2}$ \\ Max-Planck-Institut für Astrophysik, Karl-Schwarzschild-Str. 1, 85741 Garching, \\ Germany; \\ fritz@mpa-garching.mpg.de, wfh@mpa-garching.mpg.de
}

\begin{abstract}
Summary. The propagation of cellularly stabilized thermonuclear flames is investigated by means of numerical simulations. In Type Ia supernova explosions the corresponding burning regime establishes at scales below the Gibson length. The cellular flame stabilization - which is a result of an interplay between the LandauDarrieus instability and a nonlinear stabilization mechanism - is studied for the case of propagation into quiescent fuel as well as interaction with vortical fuel flows. Our simulations indicate that in thermonuclear supernova explosions stable cellular flames develop around the Gibson scale and that a deflagration-to-detonation transition is unlikely to be triggered from flame evolution effects here.
\end{abstract}

\section{Introduction}

The standard model of Type Ia supernovae (SNe Ia) describes these astrophysical events as thermonuclear explosions of white dwarf (WD) stars [7]. In our study, we refer to the specific scenario (for a review on SN Ia explosion models see [6]), where the WD consists of carbon and oxygen and the thermonuclear reaction propagates in form of a flame that starts out in the socalled "deflagration mode." That is, the flame is mediated by microphysical transport processes and its burning velocity is subsonic. One key ingredient in such a SN Ia model is the determination of the effective propagation velocity of the deflagration flame. The so-called "laminar burning speed," $s_{l}$, i.e. the propagation velocity of a planar deflagration flame, is much too low (a few percent of the sound speed in the unburned material) to explain powerful SN Ia explosions. The solution to this problem is provided by the concept of turbulent combustion. Instabilities on large scales result in the formation of a turbulent cascade, where large-scale eddies decay into smaller ones thereby transporting kinetic energy from large to small scales. Interaction of the flame with those eddies wrinkles the flame front and enlarges its surface. This is equivalent to an increase in the net fuel consumption rate and hence causes an acceleration of the flame.

Recent SN Ia explosion models on scales of the WD could show that in this way enough energy can be released to gravitationally unbind the star $[3,12]$. However, these models cannot resolve all relevant length scales down to the flame width and therefore have to rely on assumptions on the physics 
on unresolved small scales. The goal of our small-scale simulations of the propagation of the thermonuclear flame is to test those assumptions and eventually to reveal new physics that additionally needs to be included in the SN Ia models. The most significant assumptions of the large scale models by Reinecke et al. (e.g., [12]) are that the energy input into the turbulent cascade originate solely from large scales and the flame be stable on unresolved scales.

A fundamental feature of the turbulent cascade is that in a certain intermediate scale range, the turbulent velocity fluctuations decrease monotonically with smaller length scales of the eddies. This is captured by the corresponding scaling law. From that effect it is obvious, that there must exist a cutoff scale, below which flame propagation is not affected by the turbulent cascade anymore. Below this Gibson scale, the velocity fluctuations are so small, that the flame burns faster through the eddies than they can deform it. Thus the flame here propagates through "frozen turbulence." Our simulations aim on the study of effects around and below the Gibson scale. Except for the very late stages of the SN Ia explosion, the Gibson scale is well-separated from the width of the flame and the flame may safely be regarded as a discontinuity between burnt and unburned material.

\section{Theoretical Background}

What are the effects that dominate flame propagation below the Gibson scale? Here, two counteracting effects become important. The first is the LandauDarrieus (LD) instability [2,9]. It is based on a hydrodynamical effect anc would completely prevent flame stability on the scales under consideration This would have drastic impact on SN Ia models. However, there exists $\varepsilon$ second effect of purely geometrical origin [17], which balances the LD instability. Once the perturbations have grown to a critical size, former recesses of the flame front will develop into cusps, which possess propagation velocities slightly exceeding the burning speed of the rest of the front. This effect gives rise to a stable cellular shape of the flame. Therefore, the regime $\mathrm{o}$ flame propagation that will be studied in the present contribution is termec "cellular burning regime."

In connection with SN Ia explosions, this burning regime was studied by $[1,10]$ and $[13]$ demonstrated its existence by means of a full hydrodynamica simulation. The questions that arise here are: Can the cellular flame stabi. lization break down under certain conditions (e.g., low fuel densities or interaction with turbulent velocity fields)? If so, could the flame itself generatt turbulence on small scales (in contradiction to the assumption of large-scale SN Ia models) and thereby actively accelerate? This conjecture was put for. ward under the name active turbulent combustion by [11] on the basis of [8] The questions are closely connected to the search for a mechanism providing a deflagration-to-detonation transition (DDT) of the flame propagation mode 


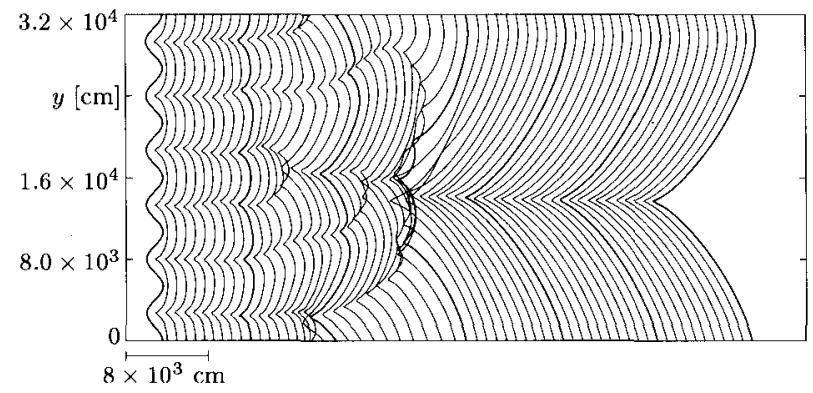

Fig. 1. Flame propagation into quiescent fuel of a density of $5 \times 10^{7} \mathrm{~g} \mathrm{~cm}^{-3}$. Each contour marks a time step of $3.3 \times 10^{-3} \mathrm{~s}$. The contours are shifted for better visibility and the spacing between them does not reflect the flame velocity.

in the context of the delayed detonation model of SNe Ia. A detonation wave is mediated by shocks and propagates with supersonic velocities.

\section{Results of Numerical Simulations}

In order to find answers to the above questions, we performed numerical simulations of the flame propagation in two dimensions. For a description and tests of the numerical implementation we refer the reader to $[13,16]$. Our simulations addressed the flame propagation into quiescent fuel as well as flame interaction with a vortical flow field. The simulations were performed on an equidistant Cartesian grid. The flame was initialized in the computational domain and perturbed in a sinusoidal way from its planar shape. To follow the long-term flame evolution, we performed the simulations in a frame of reference comoving with the flame. This was implemented by imposing an inflow boundary condition ahead of the flame front and an outflow boundary on the opposite side of the domain. Transverse to the direction of flame propagation we applied periodic boundary conditions.

\subsection{Flame Propagation into Quiescent Fuel}

The first part of our investigations addressed the flame propagation into quiescent fuel. Here two stages of flame evolution are revealed. In the beginning, the small sinusoidal perturbation imposed on the flame front increases due to the LD instability. This part of flame evolution was studied in [13], were it was shown that the growth rates observed in our simulations agree well with the expectations from Landau's linear stability analysis [9]. In the second, nonlinear stage the flame stabilizes in a cellular pattern. The propagation of a flame with an initial perturbation of 6 periods fitting into the domain is plotted in Fig. 1. 


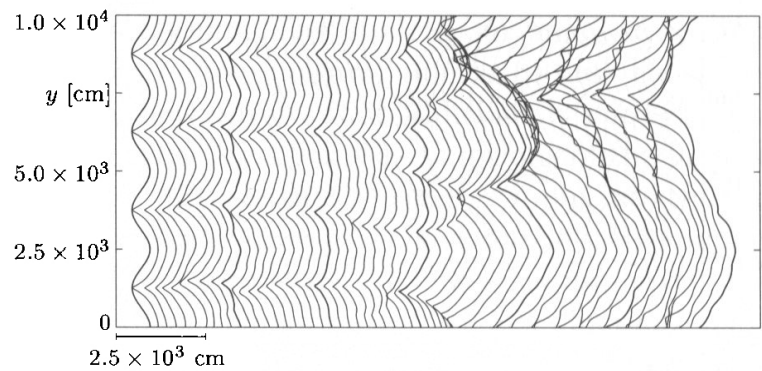

(a)

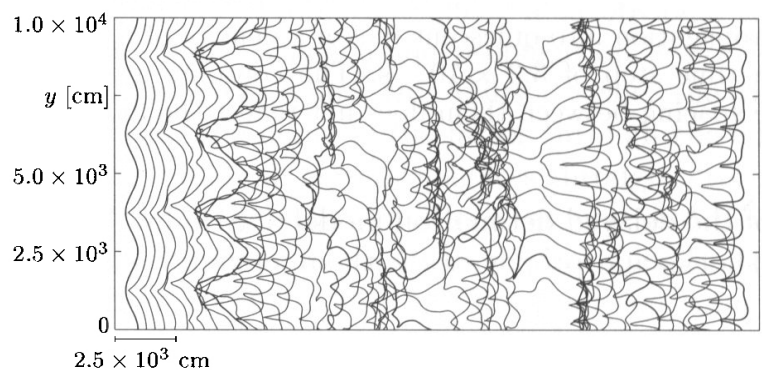

(b)

Fig. 2. Flame propagation into vortical fuel of a density of $5 \times 10^{7} \mathrm{~g} \mathrm{~cm}^{-3}$. (a) $v^{\prime} / s_{l}=0.7 ;$ (b) $v^{\prime} / s_{l}=2.5$. Each contour represents a time step of $8.0 \times 10^{-4} \mathrm{~s}(\mathrm{a})$, and $2.4 \times 10^{-3} \mathrm{~s}(\mathrm{~b})$. Again, the contours are artificially shifted for better visibility.

The tendency of the small cells to merge in the nonlinear regime finally stabilizing in a single domain-filling cusp-like structure is apparent here. This result is consistent with semi-analytical studies of flame evolution [4].

\subsection{Flame Propagation into Vortical Fuel}

In SN Ia explosions, turbulent velocity fluctuations around the Gibson scale can be expected and also relics from pre-ignition convection may contribute to turbulent flows. In order to explore the effects of flame interaction with such velocity fluctuations, we modified the inflow boundary condition to a so-called "oscillating inflow condition," which generates vortices by imposing the following velocities at the boundary

$$
\begin{aligned}
& v_{x}=s_{l}\left\{-1+v^{\prime} \sin 2 k \pi y \cos 2 k \pi\left(x-t s_{l}\right)\right\} \\
& v_{y}=s_{l} v^{\prime} \cos 2 k \pi y \sin 2 k \pi\left(x-t s_{l}\right) .
\end{aligned}
$$

Here, the parameter $v^{\prime}$ characterizes the strength of the imprinted velocity fluctuations and $k$ denotes the wavenumber of the oscillation. This produces what is termed "square vortices" by [5].

The results of two of such simulations for different strengths of the square vortices are plotted in Fig. 2 . 
Although in case of weak vortices in the incoming flow there is some interaction with the flame, the flame still shows the tendency to align in a large-wavelength cellular structure (cf. Fig. 2a). This is similar to the case of flame propagation into quiescent fuel. On the other hand, if the vortices are strong enough, they can completely break up the cellular stabilization and prevent the flame from evolving into a single domain-filling structure (cf. Fig. 2b). However, we do not observe a drastic increase in flame surface and thus flame propagation speed. The flame structure rather adapts to the vortices imprinted on the fuel flow.

\section{Conclusions}

The presented simulations of flame propagation into quiescent fuel are in good agreement with theoretical expectations. The linear stage of flame evolution is consistent with Landau's dispersion relation $[9,13]$ and in the nonlinear regime the flame stabilizes in a cellular pattern. Thus, our hydrodynamical model of flame evolution shows that the cellular burning regime exists for $\mathrm{SN}$ Ia explosions.

Interaction of the flame with vortical flow fields may lead to a break-down of cellular stabilization if the velocity fluctuations are sufficiently large. In this case, however, the flame shows the tendency to adapt to the imprinted flow field. Thus, we observe a moderate increase in the effective flame propagation velocity but no drastic effects. No indication of active turbulent combustion could be found. Therefore, it seems unlikely that effects around the Gibson scale account for a DDT at late stages of the SN Ia explosion as has been anticipated by [11]. More detailed discussions of flame propagation into quiescent fuel at different fuel densities and interaction with vortical flow fields of varying strengths are currently in preparation $[14,15]$.

\section{References}

1. S.I. Blinnikov, P.V. Sasorov: Phys. Rev. E 53, 4827 (1996)

2. G. Darrieus: In: "Propagation d'un front de flame." Presented at: La Technique Moderne, unpublished (1938)

3. V.N. Gamezo, A.M. Khokhlov, E.S. Oran, A.Y. Chtchelkanova, R.O. Rosenberg: Science 299, 77 (2003)

4. S. Gutman, G.I. Sivashinsky: Physica D 43, 129 (1990)

5. B.T. Helenbrook, C.K. Law: Combustion and Flame 117, 155 (1999)

6. W. Hillebrandt, J.C. Niemeyer: Ann. Rev. Astron. Astrophys. 38, 191 (2000)

7. F. Hoyle, W.A. Fowler: Astrophys. J. 132, 565 (1960)

8. A.R. Kerstein: Combust. Sci. Technol. 118, 189 (1996)

9. L.D. Landau: Acta Physicochim URSS 19, 77 (1944)

10. J.C. Niemeyer, W. Hillebrandt: Astrophys. J. 452, 779 (1995)

11. J.C. Niemeyer, S.E. Woosley: Astrophys. J. 475, 740 (1997) 
12. M. Reinecke, W. Hillebrandt, J.C. Niemeyer: Astron. Astrophys. 391, 1167 (2002)

13. F.K. Röpke, J.C. Niemeyer, W. Hillebrandt: Astrophys. J. 588, 952 (2003)

14. F.K. Röpke, W. Hillebrandt, J.C. Niemeyer: In: "The Cellular Burning Regime in Type Ia Supernova explosions: I. Flame Propagation into Quiescent Fuel." Astron. Astrophys., inpress (2004)

15. F.K. Röpke, W. Hillebrandt, J.C. Niemeyer: In: "The Cellular Burning Regime in Type Ia Supernova explosions: II. Flame Propagation into Vortical Fuel." Astron. Astrophys., inpress (2004)

16. F.K. Röpke: In: "On the Stability of Thermonuclear Flames in Type Ia Supernova Explosions." Ph.D. Thesis (Technical University of Munich: 2003)

17. Ya.B. Zel'dovich: J. Appl. Mech. and Tech. Phys. 1, 68 (1966) 